\title{
BOUNDS FOR HARDY DIFFERENCES
}

\author{
SABIR HUSSAIN ${ }^{\otimes 1}$ and JOSIP PEČARIĆ 2,3
}

(Received 22 February, 2008; revised 13 March, 2008)

\begin{abstract}
Bounds for Hardy differences, that is, improvements and reverses of the well-known Hardy inequality, are obtained.

2000 Mathematics subject classification: primary 26D15; secondary 26A51.

Keywords and phrases: logarithmic convexity, Boas inequality, Hardy inequality.
\end{abstract}

\section{Introduction}

Hardy [3] announced and then proved [4] a highly important classical integral inequality,

$$
\int_{0}^{\infty}\left(\frac{1}{x} \int_{0}^{x} f(t) d t\right)^{p} d x \leq\left(\frac{p}{p-1}\right)^{p} \int_{0}^{\infty}[f(x)]^{p} d x,
$$

the so-called Hardy inequality, where $p>1$ and $f \in L^{p}(0, \infty)$ is a nonnegative function. There are many extensions of (1.1) [1, 5, 6]. Boas [2] (see also Pečarić et al. [7, p. 225]) proved the following result.

THEOREM 1.1. Let $\phi$ be a continuous convex function. Let $f$ be a measurable and nonnegative function, $\lambda$ an increasing and bounded function, and let $L=\lambda(\infty)-\lambda(0)$. Then

$$
\int_{0}^{\infty} x^{-1} \phi\left(L^{-1} \int_{0}^{\infty} f(u x) d \lambda(u)\right) d x \leq \int_{0}^{\infty} x^{-1} \phi(f(x)) d x .
$$

If $\phi$ is a continuous concave function, then the inequality (1.2) holds in reverse order.

\footnotetext{
${ }^{1}$ Institute of Space Technology, Near Rawat Tool Plaza, Islamabad Highway, Islamabad, Pakistan; e-mail: sabirhus@gmail.com.

${ }^{2}$ Abdus Salam School of Mathematical Sciences, GC University, Lahore, Pakistan.

${ }^{3}$ University of Zagreb, Faculty of Textile Technology, 10000 Zagreb, Croatia;

e-mail: pecaric@mahazu.hazu.hr.

(c) Australian Mathematical Society 2011, Serial-fee code 1446-1811/2011\$16.00
} 
As a special case, Boas obtained the following extension of (1.1) (as well as some inequalities similar to (1.1)) [7, p. 230]:

$$
\int_{0}^{\infty} x^{-k}\left(\int_{0}^{x} g(t) d t\right)^{p} d x \leq\left(\frac{p}{k-1}\right)^{p} \int_{0}^{\infty} x^{p-k}[g(x)]^{p} d x
$$

where $k>1, p>1$ or $k<1, p<0$;

$$
\int_{0}^{\infty} x^{-k}\left(\int_{x}^{\infty} g(t) d t\right)^{p} d x \leq\left(\frac{p}{1-k}\right)^{p} \int_{0}^{\infty} x^{p-k}[g(x)]^{p} d x,
$$

where $k<1, p>1$ or $k>1, p<0$;

$$
\int_{0}^{\infty} x^{-k}\left(\int_{0}^{x} g(t) d t\right)^{p} d x \geq\left(\frac{p}{1-k}\right)^{p} \int_{0}^{\infty} x^{p-k}[g(x)]^{p} d x
$$

where $0<p<1$ and $k<1$;

$$
\int_{0}^{\infty} x^{-k}\left(\int_{x}^{\infty} g(t) d t\right)^{p} d x \geq\left(\frac{p}{k-1}\right)^{p} \int_{0}^{\infty} x^{p-k}[g(x)]^{p} d x,
$$

where $0<p<1$ and $k>1$. Here $g \in L^{p}(0, \infty)$ is a nonnegative function.

In this paper we derive some improvements and reverses of these results.

\section{Log-convexity of Boas differences}

Lemma 2.1. Define the function

$$
\varphi_{s}(x)= \begin{cases}\frac{x^{s}}{s(s-1)} & \text { for } s \neq 0,1 \\ -\log x & \text { for } s=0 \\ x \log x & \text { for } s=1 .\end{cases}
$$

Then $\varphi_{s}(x)$ is convex for $x>0$.

The following lemma is equivalent to the definition of convex function [7, p. 2].

Lemma 2.2. If $\phi$ is continuous and convex for all $s_{1}, s_{2}, s_{3}$ in an open interval I such that $s_{1}<s_{2}<s_{3}$, then

$$
\phi\left(s_{1}\right)\left(s_{3}-s_{2}\right)+\phi\left(s_{2}\right)\left(s_{1}-s_{3}\right)+\phi\left(s_{3}\right)\left(s_{2}-s_{1}\right) \geq 0 .
$$

We quote another useful lemma from log-convexity theory.

Lemma 2.3 (Simic [8]). A positive function $f$ is log-convex in the Jensen sense on an open interval I, that is, for each $s, t \in I$,

$$
f(s) f(t) \geq f^{2}\left(\frac{s+t}{2}\right)
$$


if and only if the relation

$$
u^{2} f(s)+2 u w f\left(\frac{s+t}{2}\right)+w^{2} f(t) \geq 0
$$

holds for each real $u, w$ and $s, t \in I$.

We now prove log-convexity of the Boas difference, that is, the difference between the expressions on either side of (1.2).

Theorem 2.4. Let the conditions of Theorem 1.1 be satisfied and let $F: \mathbb{R} \rightarrow \mathbb{R}_{+}$be a function defined by

$$
F(s)=\int_{0}^{\infty} x^{-1} \varphi_{s}(f(x)) d x-\int_{0}^{\infty} x^{-1} \varphi_{s}\left(L^{-1} \int_{0}^{\infty} f(u x) d \lambda(u)\right) d x
$$

Then $F(s)$ is log-convex, that is,

$$
[F(p)]^{r-s} \leq[F(r)]^{p-s}[F(s)]^{r-p}
$$

for $-\infty<s<p<r<\infty$.

Proof. Let us consider the function $\phi$ defined by

$$
\phi(x)=u^{2} \varphi_{s}(x)+2 u w \varphi_{r}(x)+w^{2} \varphi_{p}(x) \quad \text { where } r=\frac{s+p}{2} \text { and } u, w \in \mathbb{R} .
$$

Then

$$
\phi^{\prime \prime}(x)=u^{2} x^{s-2}+2 u w x^{r-2}+w^{2} x^{p-2}=\left(u x^{s / 2-1}+w x^{p / 2-1}\right)^{2} \geq 0 \quad \text { for } x>0 .
$$

The function $\phi$ is convex for $x>0$; therefore (1.2) is equivalent to

$$
u^{2} F(s)+2 u w F(r)+w^{2} F(p) \geq 0,
$$

that is, by Lemma 2.3,

$$
[F(r)]^{2} \leq F(s) F(p) .
$$

So $F$ is log-convex in the Jensen sense. Since

$$
\lim _{s \rightarrow 0} F(s)=F(0) \quad \text { and } \quad \lim _{s \rightarrow 1} F(s)=F(1),
$$

$F$ is continuous for $s \in \mathbb{R}$ and therefore $\log F$ is convex.

Lemma 2.2 for $-\infty<s<p<r<\infty$ yields

$$
(r-s) \log F(p) \leq(r-p) \log F(s)+(p-s) \log F(r),
$$

which is equivalent to (2.2). 


\section{Improvement and reverse of the Hardy inequality}

We obtain an improvement and reverse of the Hardy inequality and of its dual inequality.

Theorem 3.1. Let $\varphi_{s}$ and $F$ be given by Lemma 2.1 and Theorem 2.4, respectively. Let $g \in L^{1}(0, \infty)$ be a nonnegative function. Then, for $p \in \mathbb{R} \backslash\{0,1\}$,

$$
\begin{aligned}
& \frac{1}{p(p-1)}\left\{\left(\frac{p}{k-1}\right)^{p} \int_{0}^{\infty} x^{p-k}[g(x)]^{p} d x-\int_{0}^{\infty} x^{-k}\left(\int_{0}^{x} g(t) d t\right)^{p} d x\right\} \\
& \leq\left(\frac{p}{k-1}\right)^{p}[H(s)]^{(r-p) /(r-s)}[H(r)]^{(p-s) /(r-s)}
\end{aligned}
$$

for $-\infty<s<p<r<\infty$, and

$$
\begin{aligned}
& \frac{1}{p(p-1)}\left\{\left(\frac{p}{k-1}\right)^{p} \int_{0}^{\infty} x^{p-k}[g(x)]^{p} d x-\int_{0}^{\infty} x^{-k}\left(\int_{0}^{x} g(t) d t\right)^{p} d x\right\} \\
& \geq\left(\frac{p}{k-1}\right)^{p}[H(s)]^{(r-p) /(r-s)}[H(r)]^{(p-s) /(r-s)}
\end{aligned}
$$

for $-\infty<r<s<p<\infty$ and $-\infty<p<r<s<\infty$, where

$$
\begin{aligned}
H(r)= & \int_{0}^{\infty} x^{-1} \varphi_{r}\left(x^{(p-k+1) / p} g(x)\right) d x \\
& -\int_{0}^{\infty} x^{-1} \varphi_{r}\left(\frac{k-1}{p} x^{(1-k) / p} \int_{0}^{x} g(t) d t\right) d x .
\end{aligned}
$$

Proof. For $\alpha>0$, let $\lambda(u)$ be defined by

$$
\lambda(u)= \begin{cases}\alpha^{-1} u^{\alpha} & \text { for } 0 \leq u \leq 1 \\ \alpha^{-1} & \text { for } u>1\end{cases}
$$

In this case (2.1) becomes

$$
\begin{aligned}
F_{\alpha}(s) & =\int_{0}^{\infty} x^{-1} \varphi_{s}(f(x)) d x-\int_{0}^{\infty} x^{-1} \varphi_{s}\left(\alpha \int_{0}^{1} f(u x) d\left(\frac{u^{\alpha}}{\alpha}\right)\right) d x \\
& =\int_{0}^{\infty} x^{-1} \varphi_{s}(f(x)) d x-\int_{0}^{\infty} x^{-1} \varphi_{s}\left(\alpha \int_{0}^{1} f(u x) u^{\alpha-1} d u\right) d x
\end{aligned}
$$

Put $t=u x$, so that $d t=x d u$. For $0 \leq u \leq 1$ we have $0 \leq t \leq x$, that is,

$$
\begin{aligned}
F_{\alpha}(s) & =\int_{0}^{\infty} x^{-1} \varphi_{s}(f(x)) d x-\int_{0}^{\infty} x^{-1} \varphi_{s}\left(\alpha \int_{0}^{x} f(t)\left(\frac{t}{x}\right)^{\alpha-1} \frac{d t}{x}\right) d x \\
& =\int_{0}^{\infty} x^{-1} \varphi_{s}(f(x)) d x-\int_{0}^{\infty} x^{-1} \varphi_{s}\left(\alpha x^{-\alpha} \int_{0}^{x} t^{\alpha-1} f(t) d t\right) d x .
\end{aligned}
$$


For this function, inequality (2.2) becomes

$$
\left[F_{\alpha}(p)\right]^{r-s} \leq\left[F_{\alpha}(r)\right]^{p-s}\left[F_{\alpha}(s)\right]^{r-p},
$$

that is, $F_{\alpha}(p)$ is log-convex.

Now put $\alpha=(k-1) / p$ (for $p \neq 0)$ and $f(t)=t^{1-\alpha} g(t)$ in (3.3) to obtain

$$
\begin{aligned}
& \int_{0}^{\infty} x^{-1} \varphi_{p}\left(x^{(p-k+1) / p} g(x)\right) d x-\int_{0}^{\infty} x^{-1} \varphi_{p}\left(\frac{k-1}{p} x^{(1-k) / p} \int_{0}^{x} g(t) d t\right) d x \\
& \leq[H(s)]^{(r-p) /(r-s)}[H(r)]^{(p-s) /(r-s)}
\end{aligned}
$$

for $-\infty<s<p<r<\infty$. From (3.4) for $p \in \mathbb{R} \backslash\{0,1\}$ we obtain (3.1).

If in (3.3) $s \rightarrow r, p \rightarrow s, r \rightarrow p$ and $s \rightarrow p, p \rightarrow r, r \rightarrow s$, then

$$
\left[F_{\alpha}(p)\right]^{s-r} \geq\left[F_{\alpha}(r)\right]^{s-p}\left[F_{\alpha}(s)\right]^{p-r} .
$$

Put $\alpha=(k-1) / p$ and $f(t)=t^{\alpha-1} g(t)$ to obtain

$$
\begin{aligned}
& \int_{0}^{\infty} x^{-1} \varphi_{p}\left(x^{(p-k+1) / p} g(x)\right) d x-\int_{0}^{\infty} x^{-1} \varphi_{p}\left(\frac{k-1}{p} x^{(1-k) / p} \int_{0}^{x} g(t) d t\right) d x \\
& \geq[H(s)]^{(r-p) /(r-s)}[H(r)]^{(p-s) /(r-s)},
\end{aligned}
$$

from which for $p \in \mathbb{R} \backslash\{0,1\}$ we obtain (3.2).

REMARK 3.2. We have in fact proved a more general result, namely that (3.4) is valid for $-\infty<s<p<r<\infty$, and (3.5) for $-\infty<r<s<p<\infty$ and $-\infty<p<r<s<\infty$.

The following result is the dual to Theorem 3.1.

Theorem 3.3. Let $\varphi_{s}$ and $F$ be given by Lemma 2.1 and Theorem 2.4, respectively. Let $g \in L^{1}(0, \infty)$ be a nonnegative function. Then, for $p \in \mathbb{R} \backslash\{0,1\}$,

$$
\begin{aligned}
& \frac{1}{p(p-1)}\left\{\left(\frac{p}{1-k}\right)^{p} \int_{0}^{\infty} x^{p-k}[g(x)]^{p} d x-\int_{0}^{\infty} x^{-k}\left(\int_{x}^{\infty} g(t) d t\right)^{p} d x\right\} \\
& \leq\left(\frac{p}{1-k}\right)^{p}[\tilde{H}(s)]^{(r-p) /(r-s)}[\tilde{H}(r)]^{(p-s) /(r-s)}
\end{aligned}
$$

for $-\infty<s<p<r<\infty$, and

$$
\begin{aligned}
& \frac{1}{p(p-1)}\left\{\left(\frac{p}{1-k}\right)^{p} \int_{0}^{\infty} x^{p-k}[g(x)]^{p} d x-\int_{0}^{\infty} x^{-k}\left(\int_{x}^{\infty} g(t) d t\right)^{p} d x\right\} \\
& \geq\left(\frac{p}{1-k}\right)^{p}[\tilde{H}(s)]^{(r-p) /(r-s)}[\tilde{H}(r)]^{(p-s) /(r-s)}
\end{aligned}
$$

for $-\infty<r<s<p<\infty$ and $-\infty<p<r<s<\infty$, where

$$
\begin{aligned}
\tilde{H}(r)= & \int_{0}^{\infty} x^{-1} \varphi_{r}\left(x^{(p-k+1) / p} g(x)\right) d x \\
& -\int_{0}^{\infty} x^{-1} \varphi_{r}\left(\frac{1-k}{p} x^{(1-k) / p} \int_{x}^{\infty} g(t) d t\right) d x
\end{aligned}
$$


Proof. For $\beta>0$, let $\lambda(u)$ be defined by

$$
\lambda(u)= \begin{cases}0 & \text { for } 0 \leq u \leq 1 \\ \beta^{-1}\left(1-u^{-\beta}\right) & \text { for } u>1\end{cases}
$$

In this case (2.1) becomes

$$
\begin{aligned}
F_{\beta}(s) & =\int_{0}^{\infty} x^{-1} \varphi_{s}(f(x)) d x-\int_{0}^{\infty} x^{-1} \varphi_{s}\left(\int_{1}^{\infty} f(u x) d\left(1-u^{-\beta}\right)\right) d x \\
& =\int_{0}^{\infty} x^{-1} \varphi_{s}(f(x)) d x-\int_{0}^{\infty} x^{-1} \varphi_{s}\left(\beta \int_{1}^{\infty} f(u x) u^{-\beta-1} d u\right) d x
\end{aligned}
$$

Put $t=u x$, so that $d t=x d u$. For $u>1$ we have $t>x$, that is,

$$
\begin{aligned}
F_{\beta}(s) & =\int_{0}^{\infty} x^{-1} \varphi_{s}(f(x)) d x-\int_{0}^{\infty} x^{-1} \varphi_{s}\left(\beta \int_{x}^{\infty} f(t)\left(\frac{t}{x}\right)^{1+\beta} \frac{d t}{x}\right) d x \\
& =\int_{0}^{\infty} x^{-1} \varphi_{s}(f(x)) d x-\int_{0}^{\infty} x^{-1} \varphi_{s}\left(\beta x^{\beta} \int_{x}^{\infty} t^{-\beta-1} f(t) d t\right) d x .
\end{aligned}
$$

For this function, inequality (2.2) becomes

$$
\left[F_{\beta}(p)\right]^{r-s} \leq\left[F_{\beta}(r)\right]^{p-s}\left[F_{\beta}(s)\right]^{r-p},
$$

that is, $F_{\beta}(p)$ is log-convex.

Now put $\beta=(1-k) / p$ (for $p \neq 0)$ and $f(t)=t^{1+\beta} g(t)$ in (3.8) to obtain

$$
\begin{aligned}
& \int_{0}^{\infty} x^{-1} \varphi_{p}\left(x^{(p-k+1) / p} g(x)\right) d x-\int_{0}^{\infty} x^{-1} \varphi_{p}\left(\frac{1-k}{p} x^{(1-k) / p} \int_{x}^{\infty} g(t) d t\right) d x \\
& \quad \leq[\tilde{H}(s)]^{(r-p) /(r-s)}[\tilde{H}(r)]^{(p-s) /(r-s)}
\end{aligned}
$$

for $-\infty<s<p<r<\infty$. From (3.9) for $p \in \mathbb{R} \backslash\{0,1\}$ we obtain (3.6).

If in (3.8) $s \rightarrow r, p \rightarrow s, r \rightarrow p$ and $s \rightarrow p, p \rightarrow r, r \rightarrow s$, then

$$
\left[F_{\beta}(p)\right]^{s-r} \geq\left[F_{\beta}(r)\right]^{s-p}\left[F_{\beta}(s)\right]^{p-r} .
$$

Put $\beta=(1-k) / p$ and $f(t)=t^{1+\beta} g(t)$ to obtain

$$
\begin{aligned}
& \int_{0}^{\infty} x^{-1} \varphi_{p}\left(x^{(p-k+1) / p} g(x)\right) d x-\int_{0}^{\infty} x^{-1} \varphi_{p}\left(\frac{1-k}{p} x^{(1-k) / p} \int_{x}^{\infty} g(t) d t\right) d x \\
& \quad \geq[\tilde{H}(s)]^{(r-p) /(r-s)}[\tilde{H}(r)]^{(p-s) /(r-s)},
\end{aligned}
$$

from which for $p \in \mathbb{R} \backslash\{0,1\}$ we obtain (3.7).

REMARK 3.4. We have in fact proved a more general result, namely that (3.9) is valid for $-\infty<s<p<r<\infty$, and (3.10) for $-\infty<r<s<p<\infty$ and $-\infty<p<r<s<\infty$. 


\section{References}

[1] Y. Bicheng, Z. Zhuohua and L. Debnath, "On new generalizations of Hardy's integral inequality", J. Math. Anal. Appl. 217 (1998) 321-327, doi:10.1006/jmaa.1998.5758.

[2] R. P. Boas, "Some integral inequalities related to Hardy's inequality", J. Anal. Math. 23 (1970) 53-63, doi:10.1007/BF02795488.

[3] G. H. Hardy, "Note on a theorem of Hilbert", Math. Z. 6 (1920) 314-317, doi:10.1007/BF01199965.

[4] G. H. Hardy, "Notes on some points in the integral calculus, LX: an inequality between integrals (60)", Messenger Math. 54 (1925) 150-156.

[5] C. O. Imoru, "On some extensions of Hardy's inequality", Int. J. Math. Math. Sci. 8 (1995) 165-171, doi:10.1155/S0161171285000151.

[6] M. Izumi, S. Izumi and G. Peterson, "On Hardy's inequality and its generalization", Tohoku Math. J. 21 (1999) 601-613, doi:10.2748/tmj/1178242904.

[7] J. E. Pečarić, F. Proschan and Y. L. Tong, Convex functions, partial orderings, and statistical applications (Academic Press, San Diego, CA, 1992).

[8] S. Simic, "On logarithmic convexity for differences of power means", J. Inequal. Appl. 2007 (2007) Article ID 37359, 8 pages, doi:10.1155/2007/37359. 